\title{
Effectiveness of Signal Classification using Chernoff and Kailath Boundary Conditions
}

\author{
Leonid G. Dorosinsky ${ }^{1, \text { a) }}$, Nina S. Vinogradova ${ }^{1, b)}$ \\ ${ }^{1}$ Yeltsin Ural Federal University, Yekaterinburg, Russia \\ a) Corresponding author: n.s.vinogradova@urfu.ru \\ b)1.g.dorosinsky@urfu.ru
}

\begin{abstract}
The probabilities calculation of correct and incorrect surface changes recognition observed by radars placed on satellites is an actual task. In the paper, analytical expressions for calculating the efficiency of classification based on the Chernoff and Kailath boundary ratios, are obtained. The expressions are illustrated by a number of practically useful examples of detecting and recognizing the changes in the radar image.
\end{abstract}

Keywords: radar image, Chernoff boundary ratio, Kailath boundary ratio, class recognition.

\section{INTRODUCTION}

Classification of spatially distributed targets (SDT) is the foundation for the adoption of important production, environmental, social, and other tasks in various branches of industry and agriculture. Examples include such tasks as: forecasting the ice conditions in the sea and ocean surface, monitoring emergency situations (fires, landslides, floods), detecting unauthorized logging of forest areas, crops forecasts, and so on. Probabilities determining the correct and incorrect recognition of classes of SDT (using the analytical expressions and statistical simulation) may have both independent significances in the developing of a recognition system, so, they are the foundation for a choice reason of the most important tactical and technical parameters of the radar system, for example, those may be the energy potential and spatial resolution from the standpoint of required quality of the device as a whole. The problem of efficiency classification analyzing includes solving three main problems. First for, it is a methodology development for calculation potential classification accuracy. Secondly, we need to obtain the calculating expression for determining the probabilities of incorrect and correct decisions. This can be done by using the vector of the observable data and correct decision on the radar image from the SDT. Note that those one obtained as a result of the primary processing on the basis of the synthetic aperture radar. And finally, the statistical simulation of the classification should be implemented. Desirable are classifications with the quasi optimal and good empirical features.

\section{ASSESSMENT OF THE EFFECTIVENESS OF THE SDT CLASSIFICATION USING THE CHERNOFF AND KAYLATH BOUNDARY RATIOS}

Possibilities of solving the multi-alternative hypothesis test may be described by the probabilities of making the correct $\left(P_{\text {cor }}\right)$ and incorrect $\left(P_{\text {inc }}\right)$ decisions in most practical cases. The exact analytical calculation of these probabilities may be made in the simplest particular cases only. Computer simulation is practically the only method for efficiency assessing of recognition in the most real-world radar situations. But often it is necessary to evaluate the general possibility for the functioning of the designed algorithm and make the choice of the radar system main parameter. But if to use even extremely flexible mathematical simulation, it can lead to the unacceptable expenditure of computer time due to an extremely large number of analyzing values. It is most appropriate to use Chernoff and

International Conference of Numerical Analysis and Applied Mathematics ICNAAM 2019 AIP Conf. Proc. 2293, 140022-1-140022-5; https://doi.org/10.1063/5.0027276 Published by AIP Publishing. 978-0-7354-4025-8/\$30.00 
Kaylath boundary relations. Under these conditions, for obtaining quantitative estimates of the probabilistic characteristics of the system. The probability of making the incorrect decision, while testing $(M+1)$ hypotheses [2], is

$$
P_{\mathrm{inc}}=\sum_{i=1}^{M+1} \sum_{j=i+1}^{M+1}\left[p_{i} p(j / i)+p_{j} p(i / j)\right],
$$

where $p_{i}$ is a priori probability of the $i$-th hypothesis, $p(j / i)$ is the probability of decision in favour of the $j$-th hypothesis in the case the $i$-th one is true. In practice, it would be advisable to find not exact expressions for error (1), but an estimate of the upper and lower bounds of the correct classification owing to the significant number of hypotheses. The upper limit of the error probability (1) (while testing many hypotheses) is determined by the expression

$$
P_{\mathrm{inc}} \leq \sum_{i=1}^{M+1} \sum_{j=i+1}^{M+1}\left[p_{i} C h_{i j}^{F}+p_{j} C h_{i j}^{M}\right]
$$

where $C h_{i j}^{F}, C h_{i j}^{M}$ are the Chernoff boundaries for the type I and II errors probability during the pair $i$ and $j$ of hypotheses testing with regardless of the others. The lower boundary of this probability is given by the following inequality:

$$
P_{\mathrm{inc}} \geq \sum_{i=1}^{M+1} P_{i} P_{2}(j / i), i \neq j
$$

According to [3] in general case formula (3) may be expressed as

$$
P_{\text {inc }} \geq \frac{1}{M} \sum_{i=1}^{M} \sum_{j=i+1}^{M+1} K l_{i j}
$$

The $K l_{i j}$ is the generalization of Kailath's boundaries for $i$ and $j$ hypotheses, which do not form a complete group of events

$$
K l_{i j}=0.5\left\{P_{i}+P_{j}-{\left.\sqrt{\left(P_{i}+P_{j}\right)^{2}-4\left[\left(P_{i} P_{j}\right)^{1 / 2} \exp \left(\mu_{i j}(0.5)\right)\right.}\right]^{2}}^{2},\right.
$$

where $\mu_{i j}(s)$ is the moment generating function. The above calculations are obtained for the case when the observed data is a scalar. But all results are valid if the observed data have the vector form. This change affects only the expression for the moment generating function

$$
\mu_{i j}(s)=\ln \left(\int_{-\infty}^{\infty}\left(W_{j}(U)\right)^{s}\left(W_{i}(U)\right)^{1-s} d U\right) .
$$

Let us use the method proposed in [5] to calculate the Chernoff boundaries. The method based on the Edgeworth series expansion of the probability density function assumed to be close to the Gaussian one. Restricting the first two terms of the expansion, we get

$$
C h_{i j}^{F}=C h_{i j}^{F 1}-C h_{i j}^{F 2} \frac{\gamma_{3}}{6}, C h_{i j}^{M}=C h_{i j}^{M 1}-C h_{i j}^{M 2} \frac{\gamma_{3}}{6},
$$

where

$$
\begin{gathered}
C h_{i j}^{F 1}=\exp \left[\mu_{i j}(s)-s \dot{\mu}_{i j}(s)\right] I_{0}\left[s \sqrt{\ddot{\mu}_{i j}(s)}\right], \\
C h_{i j}^{M 1}=\exp \left[\mu_{i j}(s)-(1-s) \dot{\mu}_{i j}(s)\right] I_{0}\left[(1-s) \sqrt{\ddot{\mu}_{i j}(s)}\right], \\
C h_{i j}^{F 2}=\exp \left[\mu_{i j}(s)-(1-s) \dot{\mu}_{i j}(s)\right]\left\{\left[s \sqrt{\ddot{\mu}_{i j}(s)}\right]^{3} I_{0} \sqrt{\ddot{\mu}_{i j}(s)}+(2 \pi)^{-\frac{1}{2}}\left[1-s^{2} \mu_{i j}(s)\right]\right\}, \\
C h_{i j}^{M 2}=\exp \left[\mu_{i j}(s)-(1-s) \ddot{\mu}_{i j}(s)\right]\left\{\left[(1-s)^{2} \ddot{\mu}_{i j}(s)-1\right](2 \pi)^{-\frac{1}{2}}-\left[(1-s) \sqrt{\ddot{\mu}_{i j}(s)}\right]^{3} I_{0}\left[(1-s) \sqrt{\ddot{\mu}_{i j}(s)}\right]\right\}, \\
\gamma_{3}=\mu_{i j}^{(3)}(s) \cdot\left(\ddot{\mu}_{i j}(s)\right)^{-\frac{3}{2}},
\end{gathered}
$$




$$
I(\alpha)=\exp \left(\alpha^{2} / 2\right)(2 \pi)^{-\frac{1}{2}} \int_{\alpha}^{\infty} \exp \left(-t^{2} / 2\right) d t
$$

The value of the argument $s$ in formulas (8)-(13) may be found by solving the equation

$$
\dot{\mu}_{i j}(s)=\ln \left(P_{i} / P_{j}\right) \text {. }
$$

So, for finding the upper and lower boundaries of making an erroneous solution, it is necessary to know $\mu_{i j}(s)$ and its first three derivatives. Let us use formula (6) assuming that the observable data vector $U$ is a set of the complex Gaussian samples with zero mean values. In addition, the following expression may be obtained for $\mu_{i j}(s)$ :

$$
\mu_{i j}(s)=(s-1) \ln \left(\left|R_{i}\right|\right)+s \ln \left(\left|R_{j}\right|\right)-\ln \left(\left|s R_{j}^{-1}-(1-s) R_{j}^{-1}\right|\right),
$$

where $R_{i}$ and $R_{j}$ are the correlation matrices of the $U$ vector according to the $i$ and $j$ hypotheses, respectively. Using the Kayleigh-Hamilton theorem [1,4], it could be found that $R_{j}=L_{j} L_{j}^{*}$, where $L_{j}$ is a lower triangular matrix, and the symbol ${ }^{*}$ represents Hermitian conjugation. Expression (15) may be represented as

$$
\mu_{i j}(s)=\sum_{k}\left[s \ln \lambda_{k}^{i j}-\ln \left(s \lambda_{k}^{i j}+1-s\right)\right]
$$

where $\lambda_{k}^{i j}$ is the eigenvalue of the matrix.

The first three derivatives of the $\mu_{i j}(s)$ functions are

$$
\dot{\mu}_{i j}(s)=\sum_{k}\left[\ln \lambda_{k}^{i j}-\frac{\lambda_{k}^{i j}-1}{s \lambda_{k}^{i j}+1-s}\right], \ddot{\mu}_{i j}(s)=\sum_{k}\left[\frac{\lambda_{k}^{i j}-1}{s \lambda_{k}^{i j}+1-s}\right]^{2}, \dddot{\mu}_{i j}(s)=-2 \sum_{k}\left[\frac{\lambda_{k}^{i j}-1}{s \lambda_{k}^{i j}+1-s}\right]^{3} .
$$

\section{ANALYSIS OF THE POTENTIAL CHARACTERISTICS OF THE SDT CLASSIFICATION BY AN OBSERVABLE DATA VECTOR}

The potential quality analysis of the classification procedure is made on the example of the recognition of three SDT classes, which differ each other in size and total scattering cross-section. It is considered that the SDT is a rectangle, and the value of the total scattering cross-section is evenly distributed over its surface. Let us suppose that the echo signal powers from the targets of different classes, when observed from the same angles, differ approximately in the $3 \mathrm{~dB}$. We also assume that the spatial resolution of the SAR in both directions are the same. Under this condition, the equality scales in both directions of the radar image are ensured. The following parameters are used as standards for each class: target $1(T 1)$ size is $360 \times 30 \mathrm{~m}$, target $2(T 2)$ size is $240 \times 20 \mathrm{~m}$, target $3(T 3)$ size is $120 \times 10 \mathrm{~m}$.

Figure 1a shows the dependence of the upper and lower boundaries of the correct recognition probability from the total signal-to-noise ratio for the $T 2\left(q_{2 \varepsilon}\right)$ target

$$
\begin{gathered}
P_{\text {cor }}^{\text {up }}=1-0.5 \sum_{i=1}^{2} \sum_{j=i+1}^{3} K l_{i j}, \\
P_{\text {cor }}^{\mathrm{dw}}=1-0.5 \sum_{i=1}^{2} \sum_{j=i+1}^{3}\left(P_{i} C h_{i j}^{F}+P_{j} C h_{i j}^{M}\right) .
\end{gathered}
$$

The signal-to-noise ratios for the other two goals differ on the $3 \mathrm{~dB}$

$$
q_{1 \varepsilon}=q_{2 \varepsilon}+3 d B, \quad q_{3 \varepsilon}=q_{2 \varepsilon}-3 d B .
$$

It follows from figure 1a that for reliable recognition of the targets with the probability not smaller than 0.9 , it is necessary the signal-to-noise ratio to be at least $15 \mathrm{~dB}$. It should also be noted that the classification efficiency depends on the SAR spatial resolution significantly. The figure 1,b shows the dependence of the upper and lower boundaries of correct recognition probability from spatial resolution. The signal-to-noise ratio is fixed at the 10 , 14, $18 \mathrm{~dB}$. 

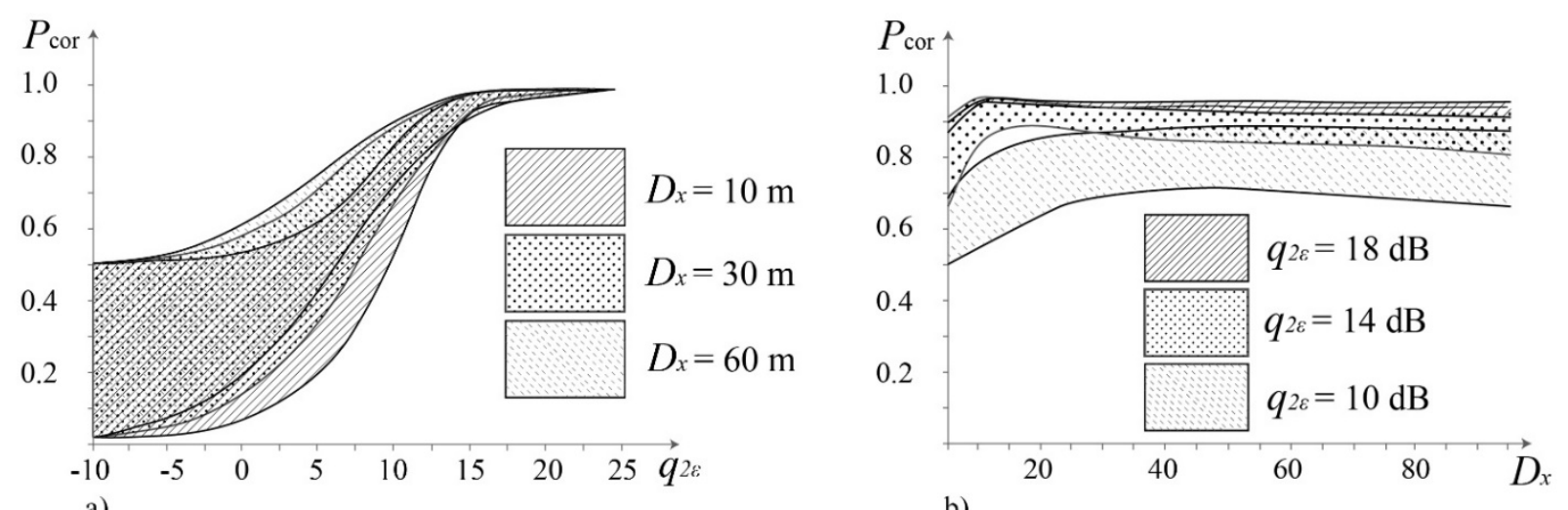

a)

b)

FIGURE 1. a) Upper and lower probability boundaries of the correct classification depending on the total signal-to-noise ratio (a second-class target 2); b) upper and lower probability boundaries of correct classification depending on resolution (for a fixed total signal-to-noise ratio); the calculations are performed at the fixed total energy of pulses.

Figure $1 \mathrm{~b}$ shows that all the curves have a maximum, which (as the signal-to-noise ratio increases) shifts to a higher spatial resolution region. It should be noted that the minimum value of $q_{2 \varepsilon}$, at which the correct recognition probability is at least 0.9 is ensured, equals to $14 \mathrm{db}$. It is recommended to select the spatial resolution in the range of 15-35 $\mathrm{m}$ in this case. As the signal-to-noise ratio increases, the resolution requirements decrease rather quickly. Thus, at the $q_{2 \varepsilon}=16 \mathrm{~dB}$ the possible values range of the required spatial resolution, it takes the interval from 6 up to $93 \mathrm{~m}$.

Let us consider the dependence of the correct recognition probability of the entire system $\left(P_{\mathrm{en}}\right)$ on the correct recognition probability of an individual spacecraft $\left(P_{\text {in }}\right)$ with a different number of spacecraft $L$. It is necessary to obtain the most general ideas about increasing the efficiency of classifying the SDT on the basis of integrating the data from different monitoring systems. The calculations are performed according to the expression

$$
P_{\mathrm{en}}=1-P_{\mathrm{in}}^{L} \text {. }
$$

The obtained dependences are shown in Fig. 2. It follows from the graphs that for obtaining the final probability more than 0.9 with using the six independent surveillance satellites, the individual probability of the correct classification by each spacecraft has to be at least 0.7 .

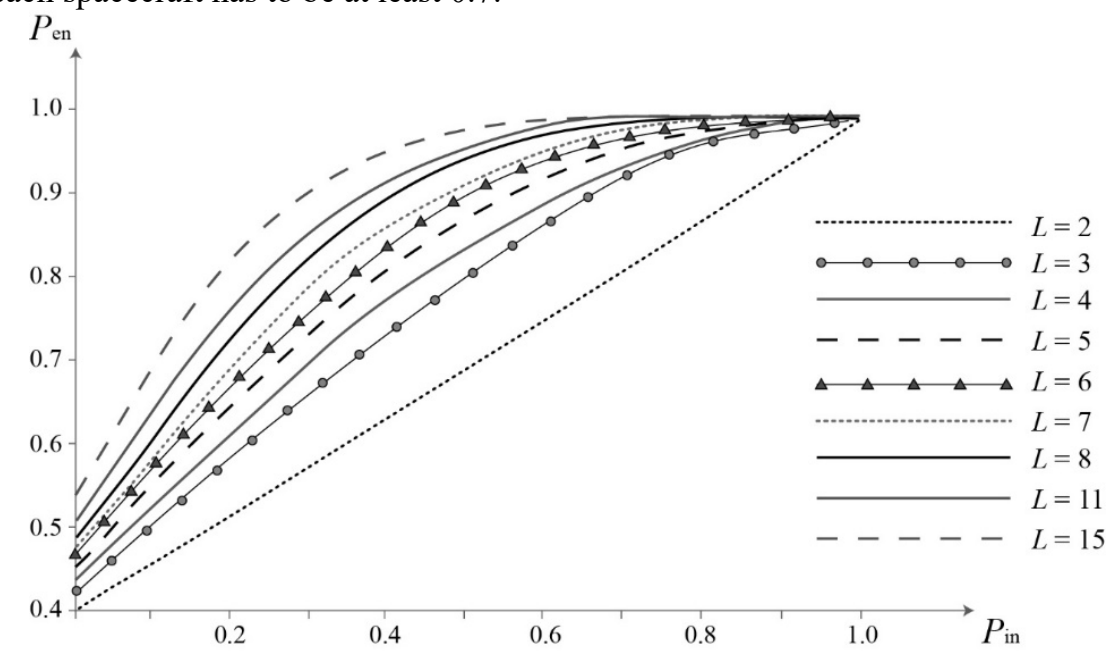

FIGURE 2. The dependences of the correct recognition probability using a simple voting on the correct recognition probability while making a decision on the individual observation. 


\section{CONCLUSION}

A method was developed for assessment of the classification potential accuracy of spatially distributed targets using the side-scan radar with synthetic aperture placed on a satellite was developed. The method is based on the complex amplitudes vector at the input of the processing system as the initial information. This makes it possible to evaluate the expected statistical characteristics (efficiency of determining the SDT class) without analyzing and taking into account the specific structure of the processing device. Therefore, it becomes possible to argue the choice of the main tactical and technical parameters of the classification system, namely, the required energy potential and spatial resolution. Analysis of the classification efficiency was made according to the proposed method for typical spatially distributed targets differing in size and specific value of total scattering cross-section. The analysis shows that to ensure satisfactory classification efficiency (probability more than 0.9) at the spatial resolution about 20-30 $\mathrm{m}$, the SDTs have to differ in size by $25-30 \%$ and in the total scattering cross-section on $3 \mathrm{~dB}$. While using a constellation of small satellites, the probability of correct classification may be significantly increased by summing the results of decisions taken by each individual satellite. For example, to obtain the resulting probability of correct classification more than 0.9 in the presence of six independent surveillance satellites, the individual probability of the correct classification of each spacecraft must be at least 0.7 .

\section{REFERENCES}

1. Bakut P.A. Bolshakov I.A. Tartakovsky G.P. The questions of the statistical radar theory (in russuan). Sovetskoe radio, Russia 424 p (1963).

2. Baumgartner, S.V., Krieger, G., Fast GMTI algorithm for traffic monitoring based on a priori knowledge. IEEE Transactions on Geoscience and Remote Sensing. vol 50, iss 11. 4626-4641 (India, 2012).

3. Fukunaga K., Introduction to statistical pattern recognition. Second Edition. Academic Press, USA, 592 p. (2013)

4. Van Tris G. Theory of detection, estimates and modulation. (in russuan). Sovetskoe radio, Russia, 344 p (1971). 